\title{
A connexin43/YAP axis regulates astroglial-mesenchymal transition in hemoglobin induced astrocyte activation
}

\author{
Yong Yang ${ }^{1,2}$ - Jie Ren ${ }^{1}$ Yuhao Sun ${ }^{1} \cdot$ Yuan Xue ${ }^{3}$ Zhijian Zhang ${ }^{4}$ - Aihua Gong ${ }^{4} \cdot$ Baofeng Wang ${ }^{1}$. \\ Zhihong Zhong ${ }^{1} \cdot$ Zhenwen Cui $^{5} \cdot$ Zhiyu Xi $^{1} \cdot$ Guo-yuan Yang ${ }^{6} \cdot$ Qingfang Sun $^{1,7} \cdot$ Liuguan Bian $^{1}$
}

Received: 25 July 2017 / Revised: 12 April 2018 / Accepted: 16 May 2018 / Published online: 7 June 2018

(c) ADMC Associazione Differenziamento e Morte Cellulare 2018

\begin{abstract}
Reactive astrogliosis is a common response to insults to the central nervous system, but the mechanism remains unknown. In this study, we found the temporal and spatial differential expression of glial fibrillary acidic protein (GFAP) and Vimentin in the intracerebral hemorrhage ( $\mathrm{ICH})$ mouse brain, indicating that the de-differentiation and astroglial-mesenchymal transition (AMT) of astrocytes might be an early event in reactive astrogliosis. Further we verified the AMT finding in purified astrocyte cultures exposed to hemoglobin $(\mathrm{Hb})$. Additionally, Connexin 43 (Cx43) downregulation and YAP nuclear translocation were observed in Hb-activated astrocytes. Knocking down $\mathrm{Cx} 43$ by siRNA triggered YAP nuclear translocation. $\mathrm{Cx} 43$ and YAP were physically associated as determined by immunofluorescence and coimmunoprecipitation. We propose that astrocytes undergo AMT during $\mathrm{Hb}$-induced activation where $\mathrm{Cx} 43$ downregulation facilitates YAP nuclear translocation is a novel mechanism involved in this process. Cx43-YAP interaction may represent a potential therapeutic target for modulating astrocyte activation.
\end{abstract}

\section{Introduction}

Intracerebral hemorrhage ( $\mathrm{ICH})$ is a serious neurological crisis accounting for $\sim 10-15 \%$ of all strokes where it is associated with high mortality and morbidity [1]. Astrocyte activation (also known as reactive astrogliosis) induced

Edited by: N. Bazan.

These authors contributed equally: Yong Yang, Jie Ren.

Electronic supplementary material The online version of this article (https://doi.org/10.1038/s41418-018-0137-0) contains supplementary material, which is available to authorized users.

Qingfang Sun

sqf10756@rjh.com.cn

$\triangle$ Liuguan Bian

blg11118@rjh.com.cn

1 Department of Neurosurgery, Ruijin Hospital, School of Medicine, Shanghai Jiao Tong University, Shanghai 200025, China

2 Department of Neurosurgery, Guangdong General Hospital, Guangdong Academy of Medical Sciences, Guangzhou 510080, China

3 Zhenjiang Center for Disease Control and Prevention, secondary injury has been reported to play a critical role in neurological deterioration [2,3]. Moreover, the resulting glial scar formation from reactive astrogliosis impedes axonal regeneration [4]. Uncovering the mechanism of astrocyte activation will help improve the patient outcomes.

Being the most dominant glial cells in the central nervous system (CNS), astrocytes provide neurotrophic and structural support to neurons and help maintain the extracellular milieu homeostasis [5-8]. Astrocyte activation is a common response to insults to the CNS including neurotrauma, infection, ischemia, hemorrhagic stroke, and neurodegeneration [9-13]. Reactive astrogliosis includes a series of continuous changes: alterations in gene and protein

\section{Zhenjiang 212000, China}

4 Basic Medical Science Research Center, School of Medicine, Jiangsu University, Zhenjiang 212000, China

5 Department of Neurosurgery, The Affiliated Hospital of Qingdao University, Qingdao 266000, China

6 Neuroscience and Neuroengineering Research Center, Med-X Research Institute, Shanghai Jiao Tong University, Shanghai 200030, China

7 Department of Neurosurgery, Ruijin Hospital Luwan Branch, Shanghai Jiao Tong University, Shanghai 200025, China 
expression, proliferation and migration, hypertrophy of the cells, glial scar formation (permanent tissue rearrangement) [14]. It was reported that cell cycle inhibition attenuated astrocyte proliferation and prevented glial scar formation [15]. So astrocyte proliferation contributes to glial scar formation. We propose that as a terminally differentiated cell, astrocytes may de-differentiate before undergoing proliferation. Our hypothesis is based, at least in part, on reports that Heparin-binding epidermal growth factor-like growth factor can induce partial de-differentiation of astrocytes in addition to promoting their proliferation [16].

Astrocytes are rich in gap junctions, which are the main form of astroglial intercellular communication. Connexin 43 $(\mathrm{Cx} 43)$ is the predominant connexin in astrocytes. It is assembled into hexamer (named connexon) and delivered to the cell membrane. The docking of neighboring connexons forms intercellular gap junction channels, which allow the direct diffusion of ions and small signaling molecules providing metabolic and electrical coupling for astrocytes [17]. This Cx43-based astroglial syncytial structure is critical for maintaining cellular homeostasis [18-22]. It is reported that in mitotic cells [23] or the malignant transformation of glioma [24], Cx43 expression is downregulated. Restoring Cx43 expression in glioma stem cells reverses their malignant phenotype by upregulating E-cadherin [25]. Here, we propose that $\mathrm{Cx} 43$ may also act as a molecular switch in astrocyte activation.

The Hippo pathway is a key regulator of tissue homeostasis. It mediates contact inhibition signaling and regulates organ size by controlling cell proliferation and stem cell expansion. In pathological conditions, Hippo signaling is highly relevant to tissue regeneration and tumor development [26]. Yes-associated protein (YAP), the pivotal effector of the Hippo pathway, acts as a transcriptional coactivator. YAP is transcriptionally active when it translocates to the nucleus and forms complexes with multiple transcription factors (TEAD, FoxO1, Smad1, etc.). The target genes of YAP-TEAD are involved in cell proliferation [27], stem cell self-renewal [28], epithelial-mesenchymal transition (EMT) [29] and anti-apoptosis [30]. The transcriptional activity of YAP is inhibited by its phosphorylation and cytoplasmic retention [31]. YAP can be sequestered at cellular adherens and tight junctions, thereby suppressing its transcriptional activity as a mechanism for cellular contact inhibition [32, 33]. YAP signaling has been implicated in astrogliogenesis and astrocytic differentiation in the developing neocortex $[34,35]$. Indeed, YAP knockout mice develop reactive astrogliosis in the cortex supporting its critical involvement in brain development [36].

Excessive cell-free hemoglobin $(\mathrm{Hb})$ released by hemolysis following ICH can be particularly problematic to extracellular homeostasis. Given their key functions, we sought to determine whether $\mathrm{Cx} 43$ and YAP are dysregulated following ICH. In particular, we sought evidence that $\mathrm{Cx} 43$-YAP cross talking can contribute to dedifferentiation and phenotype switching during ICHinduced reactive astrogliosis. To test our hypothesis, we used two complementary models of ICH or Hb injection to trace an astroglial-mesenchymal transition (AMT).

\section{Results}

\section{The astroglial-mesenchymal phenotype switching of astrocytes in ICH mouse brain}

Alterations in intermediate filaments (IF) protein expression are a recognized feature of reactive astrogliosis [37]. IF proteins are tissue specific, with glial fibrillary acidic protein (GFAP) being the hallmark for mature astrocyte and Vimentin for mesenchymal tissues. In the normal CNS, Vimentin is undetectable in mature astrocytes. Following injury, Vimentin, and GFAP expression increase markedly [38]. To test the expression pattern of GFAP and Vimentin in the context of ICH, we performed immunofluorescence staining and Western blot analysis. As shown (Fig. 1a-c), Vimentin was undetectable in normal brain, whereas after $\mathrm{ICH}$, it was intensely expressed in the hematoma margin, reaching a peak at $3 \mathrm{~d}$ and returning to normal by $7 \mathrm{~d}$ postICH when the hematoma resolved. Similarly, brain expression of GFAP significantly increased in the perilesion area at $1 \mathrm{~d}, 3 \mathrm{~d}, 7 \mathrm{~d}, 14 \mathrm{~d}$ post-ICH $(p<0.01)$. Reactive astrocytes with intensive GFAP expression and hypertrophy of cellular processes were also observed at $3 \mathrm{~d}$ and $7 \mathrm{~d}$ postICH. At $14 \mathrm{~d}$ post-ICH, glial scar formed with intensive GFAP staining. Of note, there was a transient decrease in GFAP expression at $12 \mathrm{~h}$ post-ICH $(p<0.05)$. The temporal and spatial changes in GFAP and Vimentin expression suggested a switch between astroglial and mesenchymal phenotypes during ICH-induced reactive astrogliosis.

Derived from neuroectoderm, astrocytes are considered to be specialized epithelial cells in the CNS. The AMT is analogous to the well-studied EMT. To test our AMT hypothesis, we employed E-Cadherin (epithelial marker) and N-Cadherin (mesenchymal marker) during immunofluorescence double staining tracing studies (Fig. 1d). These showed similar temporal and spatial changes in E-Cadherin and N-Cadherin expression, verifying our AMT finding.

To determine the cell type responsible for the signal of GFAP and Vimentin, we employed additional markers: Aldolase-C for astrocytes [39], NeuN for neurons, Iba-1 for microglia. The results (Fig. 1e) showed near complete overlap of signals for Aldolase- $\mathrm{C}$ and Vimentin, indicating that astrocytes were the cells responsible for AMT in vivo.

To determine whether AMT in the ICH brain can be recapitulated by $\mathrm{Hb}$, we performed additional studies with 
a
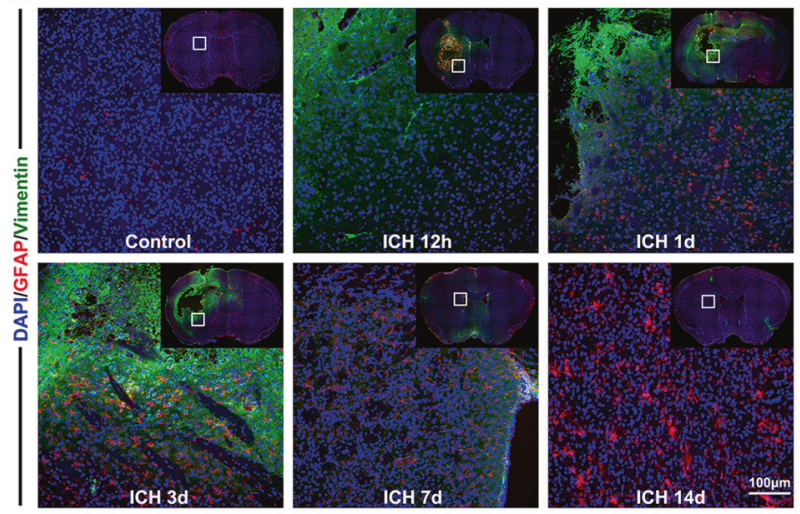

b

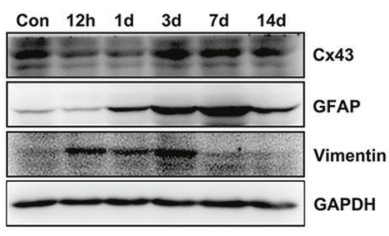

C

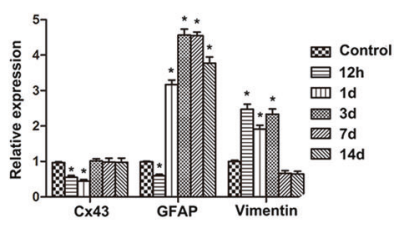

d
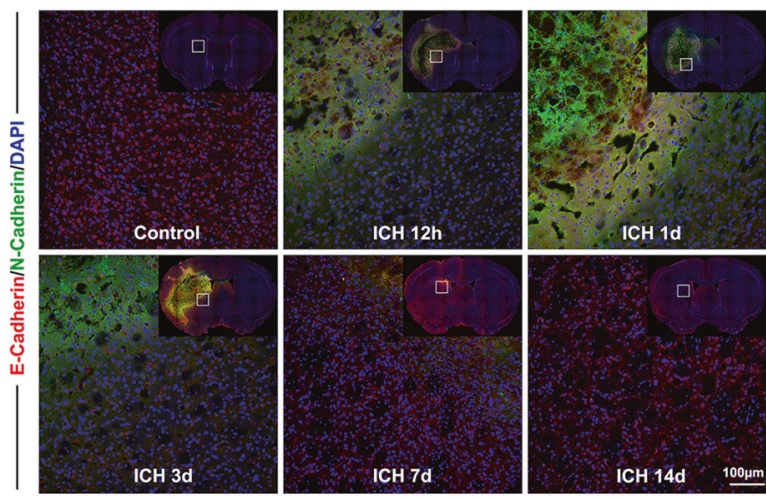

e

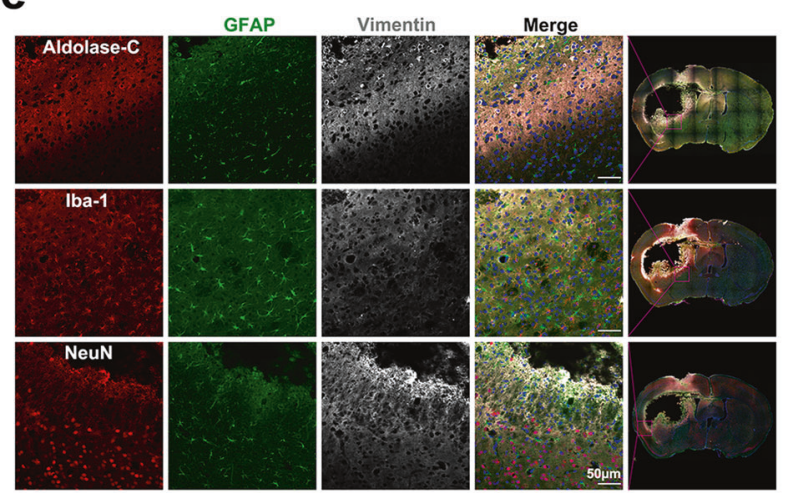

direct injection of this hematoma degradation product. Strikingly, we observed the expression of astroglial and mesenchymal markers in this Hb-injection mouse model that was highly reminiscent of what we observed in the ICH model (Figure S1 \& S2).
Fig. 1 The astroglial-mesenchymal phenotype switching of astrocytes in ICH mouse brain. a Immunofluorescence staining for Vimentin (green) and GFAP (red) in normal and ICH mouse brain at $12 \mathrm{~h}, 1 \mathrm{~d}$, $3 \mathrm{~d}, 7 \mathrm{~d}$, and $14 \mathrm{~d}$. Bar $=100 \mu \mathrm{m}$. Vimentin was undetectable in normal brain, whereas after $\mathrm{ICH}$, it was intensely expressed in the hematoma margin. The hematoma was completely resolved at $7 \mathrm{~d}$ post-ICH. The normal brain had a baseline expression of GFAP, reactive astrocytes with intensive GFAP expression and hypertrophy of cellular processes were observed in the peri-lesion area at $3 \mathrm{~d}$ and $7 \mathrm{~d}$ post-ICH. At $14 \mathrm{~d}$ post-ICH, glial scar formed with intensive GFAP staining. b Western blotting analysis of $\mathrm{Cx} 43$, GFAP and Vimentin expression in normal and ICH mouse brain at $12 \mathrm{~h}, 1 \mathrm{~d}, 3 \mathrm{~d}, 7 \mathrm{~d}$, and $14 \mathrm{~d}$. $\mathbf{c}$ The results of densitometric analysis of the bands were plotted as mean \pm SEM of five independent experiments. Vimentin expression was significantly increased after $\mathrm{ICH}$, reached a peak at $3 \mathrm{~d}$ and returned to normal at $7 \mathrm{~d}$ post-ICH. GFAP expression was significantly increased at $1 \mathrm{~d}, 3 \mathrm{~d}, 7 \mathrm{~d}$, and $14 \mathrm{~d}$ post-ICH, reached a peak at $7 \mathrm{~d}$ post-ICH. There was a transient decrease in GFAP expression at $12 \mathrm{~h}$ post-ICH. $* p<0.05, * * p<$ 0.01 compared with control. d Immunofluorescence staining for ECadherin (red) and $\mathrm{N}$-Cadherin (green) in normal and ICH mouse brain at $12 \mathrm{~h}, 1 \mathrm{~d}, 3 \mathrm{~d}, 7 \mathrm{~d}$, and $14 \mathrm{~d}$. Bar $=100 \mu \mathrm{m}$. e Immunofluorescence triple staining with Adolase-C + GFAP + Vimentin, Iba$1+$ GFAP + Vimentin, NeuN + GFAP + Vimentin in the peri-lesion area of ICH brain. Bar $=50 \mu \mathrm{m}$

\section{$\mathrm{Hb}$ induced astrocyte activation and promoted their proliferation}

From the in vivo study, we concluded that astrocytes can respond to $\mathrm{Hb}$. So we established an in vitro cellular model by exposing purified astrocyte cultures to several concentrations of Hb. IL-1 $\beta$, IL- 6 , and TNF- $\alpha$ mRNA expression were quantitated by qRT-PCR. As shown (Fig. 2a-c), cytokine expression was significantly increased after $\mathrm{Hb}$ exposure for 6 or $24 \mathrm{~h}(p<0.05)$.

We also examined astrocyte viability by the CCK- 8 assay after $\mathrm{Hb}$ exposure. The results (Fig. 2d) indicated that cell viability increased dose-dependently in the range from 0 to $30 \mu \mathrm{M}(p<0.05)$. Because $\mathrm{Hb}$ began to precipitate in the culture medium at concentrations higher than $25 \mu \mathrm{M}$, we chose $25 \mu \mathrm{M}$ as the highest concentration for subsequent experiments. Because the CCK- 8 assay reflects the activity of mitochondrial dehydrogenase rather than the actual cell number, we also detected Ki67 (a cellular marker for proliferation) expression by immunofluorescence staining to determine whether $\mathrm{Hb}$ can promote astrocyte proliferation. As shown (Fig. 2e, f), Ki67-positive cells significantly increased after $25 \mu \mathrm{M} \mathrm{Hb}$ treatment $(p<0.01)$. Taken together, these studies supported the ability of $\mathrm{Hb}$-treatment to activate and promote astrocyte proliferation.

\section{Hb-activated astrocytes underwent AMT}

To corroborate AMT in an in vitro model, we performed immunofluorescence staining for GFAP, Vimentin, and Ki67 in Hb-treated astrocyte cultures. The results (Fig. 3a) 
a
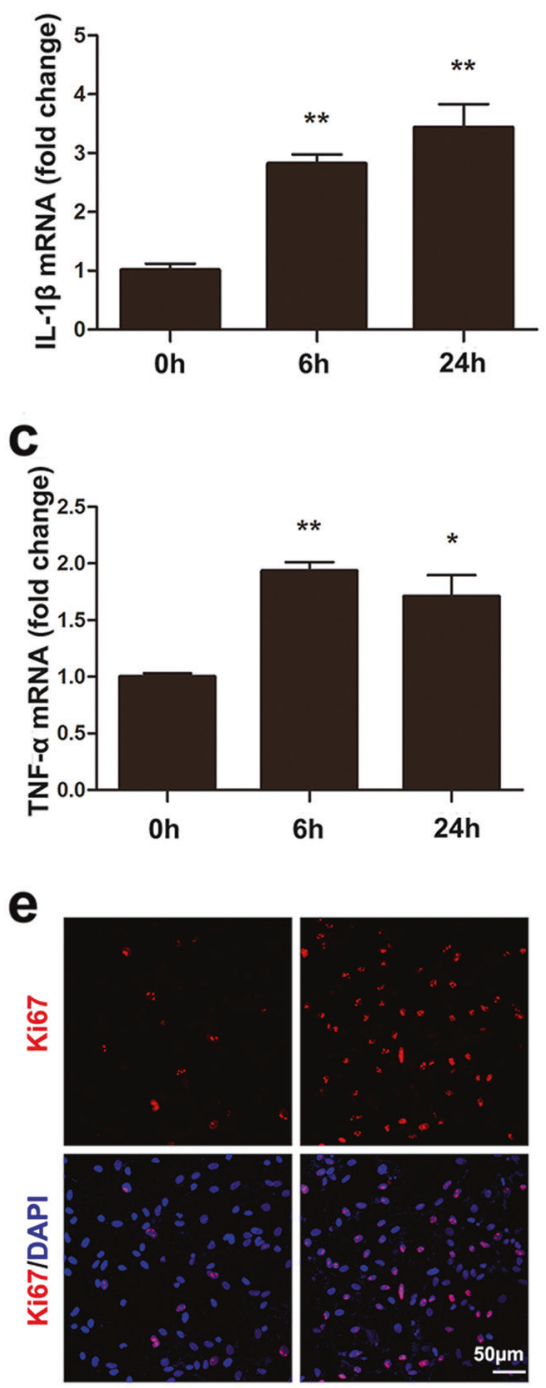

Control
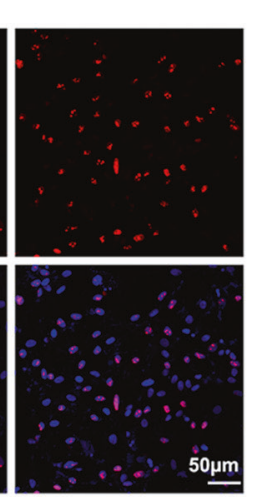

$25 \mu \mathrm{M} \mathrm{Hb}$

Fig. $2 \mathrm{Hb}$ stimulated inflammatory cytokines expression and promoted astrocytes proliferation. a-c IL- $1 \beta$, IL- 6 , and TNF- $\alpha$ mRNA expression in astrocytes treated with $25 \mu \mathrm{M} \mathrm{Hb}$ for 6 and $24 \mathrm{~h}$. mRNA expression was quantitated using real-time PCR. Data were normalized against the internal reference GAPDH. The fold change values were calculated by normalizing to control samples. The results were plotted as mean \pm SEM of three independent experiments. Hb treatment significantly increased IL-1 $\beta$, IL- 6 , and TNF- $\alpha$ mRNA expression. ${ }^{*} p<0.05,{ }^{*} p<0.01$ compared with control. d Cell viability of astrocytes treated with $0,10,20,30$ or $50 \mu \mathrm{M} \mathrm{Hb}$ for $24 \mathrm{~h}$. The

indicated a trend of astroglial-mesenchymal phenotype switching. Additionally, Western blotting analysis confirmed changes in AMT marker expression in astrocyte cultures treated with $0,10,15$, and $25 \mu \mathrm{M} \mathrm{Hb}$ for $24 \mathrm{~h}$ or treated with $25 \mu \mathrm{M} \mathrm{Hb}$ for $0,6,12$, and $24 \mathrm{~h}$. We selected GFAP and E-Cadherin as astroglial markers and Vimentin, N-Cadherin, and SLUG for mesenchymal markers. SLUG is a zinc-finger transcriptional repressor that downregulates E-cadherin expression and supports mesenchymal

b

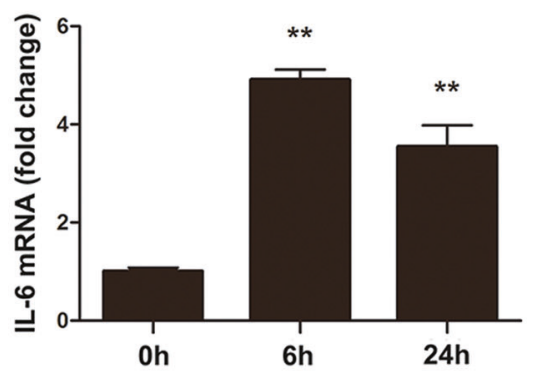

d

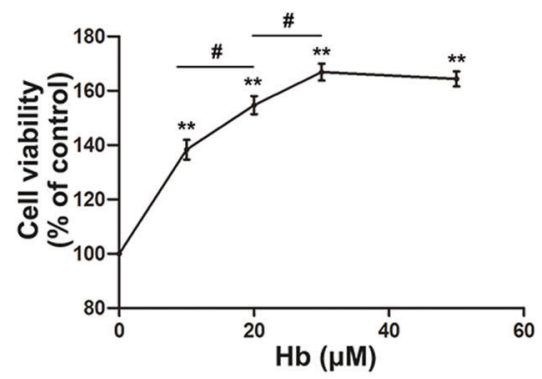

f

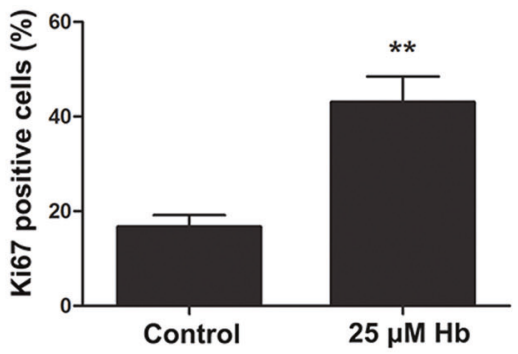

absorbance at $450 \mathrm{~nm}$ was normalized to control and expressed as percentage of control. ${ }^{* *} p<0.01$ compared with control. ${ }^{\#} p<0.05$ comparison between indicated groups. e Representative pictures of Ki67 immunofluorescence staining. Ki67-positive cells were labeled in red and the cell nuclei were counterstained with DAPI, as shown in blue. Bar $=50 \mu \mathrm{m}$. $\mathbf{f}$ The histogram showing the percentage of Ki67positive cells. The results were plotted as mean \pm SEM of three independent experiments. $\mathrm{Hb}$ treatment significantly promoted astrocyte proliferation. $* p<0.05, * * p<0.01$ compared with control

phenotype by inducing $\mathrm{N}$-Cadherin expression and eliminating apico-basal polarity [40].

The western blotting results showed that GFAP expression significantly decreased at the time point $(24 \mathrm{~h})$ (Fig. 3b, c) and concentrations (10, 15, and $25 \mu \mathrm{M}$ ) (Fig. 3d, e). E-Cadherin expression significantly decreased at the time points (12 and $24 \mathrm{~h}$ ) (Fig. 3b, c) and concentrations (10, 15, and $25 \mu \mathrm{M})$ (Fig. 3d, e). Conversely, Vimentin expression significantly increased at the time points $(6,12$, and $24 \mathrm{~h})$ 
Fig. $3 \mathrm{Hb}$ induced epithelialmesenchymal transition in astrocytes. a

Immunofluorescence staining of astrocytes for Vimentin (red), GFAP (green), and Ki67 (purple). The cell nuclei were counterstained with DAPI (blue). $\mathrm{Bar}=50 \mu \mathrm{m}$. b,d Western bloting analysis of GFAP, Vimentin, E-Cadherin, N-Cadherin, and SLUG expression in astrocytes treated with $\mathrm{Hb}$ for indicated times and doses. c,e The results of densitometric analysis of the bands were plotted as mean \pm SEM of three independent experiments. $\mathrm{Hb}$ treatment decreased GFAP and ECadherin expression and increased Vimentin, $\mathrm{N}$ Cadherin, and SLUG expression. * $p<0.05, * * p<$ 0.01 compared with control $(0 \mathrm{~h}$ or $0 \mu \mathrm{M})$
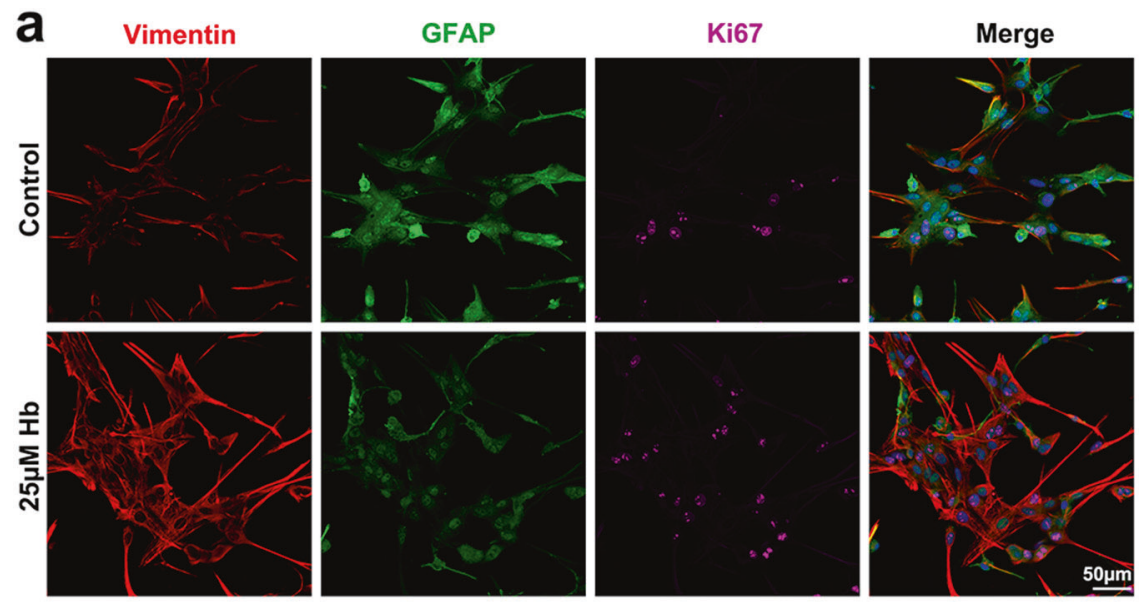

b
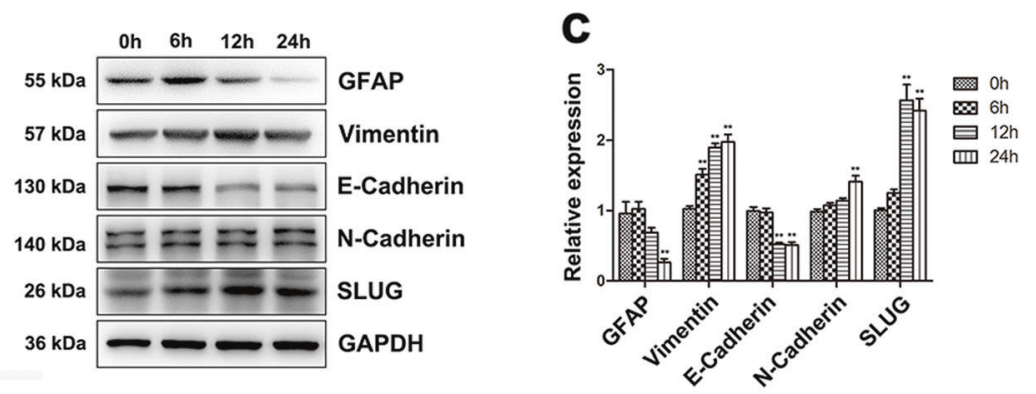

d

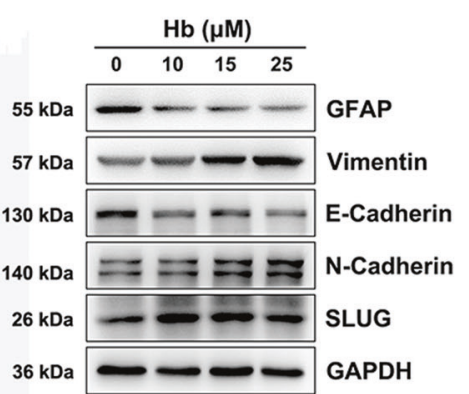

e

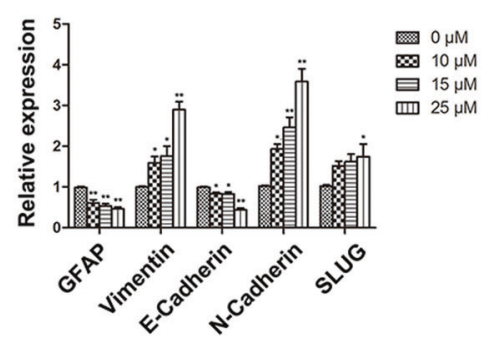

(Fig. 3b, c) and concentrations (10, 15, and $25 \mu \mathrm{M})$ (Fig. 3d, e). N-Cadherin expression significantly increased at the time point (24 h) (Fig. 3b, c) and concentrations (10, 15, and 25 $\mu \mathrm{M})$ (Fig. 3d, e). SLUG expression significantly increased at the time points (12 and $24 \mathrm{~h}$ ) (Fig. 3b, c) and concentration $(25 \mu \mathrm{M})$. (Fig. 3d, e) $(p<0.05)$. These results supported AMT during $\mathrm{Hb}$-induced astrocyte activation.

\section{$\mathrm{Hb}$ induced downregulation of astroglial Cx43}

Cx43 based intercellular gap junctions are critical for maintaining astroglial homeostasis. After ICH, the homeostasis is broken. To find out whether $\mathrm{Cx} 43$ expression will be affected, immunofluorescence staining, PCR and Western blotting analysis were performed. As shown (Fig. 1b, c), $\mathrm{Cx} 43$ expression was downregulated in mouse brain at $12 \mathrm{~h}$ and $1 \mathrm{~d}$ post-ICH $(p<0.05)$. From the immunofluorescence studies (Fig. 4a), we noted that $\mathrm{Cx} 43$ was expressed on the cell membrane where astrocytes contacted. After $\mathrm{Hb}$ treatment, $\mathrm{Cx} 43$ expression was decreased at the mRNA and protein levels. The PCR result (Fig. 4b) showed that Cx43 mRNA expression was significantly suppressed at time point $24 \mathrm{~h}(p<0.05)$. Western blotting analysis revealed that total $\mathrm{Cx} 43$ decreased significantly at 12 and $24 \mathrm{~h}(p<0.05)$, but the ratio of phosphoCx43/total Cx43 increased significantly at 3, 6, 12, and $24 \mathrm{~h}$ $(p<0.05)$ (Fig. 4c-e). These results indicated that $\mathrm{Hb}-$ treatment inhibited Cx43 mRNA transcription and induced $\mathrm{Cx} 43$ protein phosphorylation and degradation.

\section{$\mathrm{Hb}$-induced YAP nuclear translocation in astrocytes}

The Hippo pathway is a key regulator of tissue homeostasis and cellular contact inhibition, which is disrupted after ICH. 
Fig. $4 \mathrm{Hb}$ exposure induced Cx43 downregulation in astrocytes. a Immunofluorescence staining for connexin 43 (red) and GFAP (green) in astrocytes treated with or without $25 \mu \mathrm{M} \mathrm{Hb}$. The cell nuclei were counterstained with DAPI (blue). Cx43 was expressed on the cell membrane where astrocytes contact. After $\mathrm{Hb}$ treatment, $\mathrm{Cx} 43$ expression was decreased. Bar $=25 \mu \mathrm{m}$. $\mathbf{b}$ Cx43 mRNA expression in control and astrocytes treated with $25 \mu \mathrm{M} \mathrm{Hb}$ for 6 or $24 \mathrm{~h}$. Data were normalized against the internal reference GAPDH. The fold change values were calculated by normalizing to control samples. The results were plotted as mean \pm SEM of three independent experiments. $\mathrm{Hb}$ treatment for $24 \mathrm{~h}$ decreased Cx43 mRNA expression by half. $* p<0.05$ compared with control $(0 \mathrm{~h})$. c Western blotting analysis of $\mathrm{Cx} 43$ expression in astrocytes treated with $25 \mu \mathrm{M} \mathrm{Hb}$ for 0,1 , $3,6,12$, or $24 \mathrm{~h}$. d,e The results of densitometric analysis of total Cx43 and p-Cx43/total Cx43 were plotted as mean \pm SEM of three independent experiments. Hb-treatment decreased Cx 43 expression and increased the ratio of $\mathrm{p}-\mathrm{Cx} 43 /$ total $\mathrm{Cx} 43$. $* p<$ $0.05, * * p<0.01$ compared with control $(0 \mathrm{~h})$ a
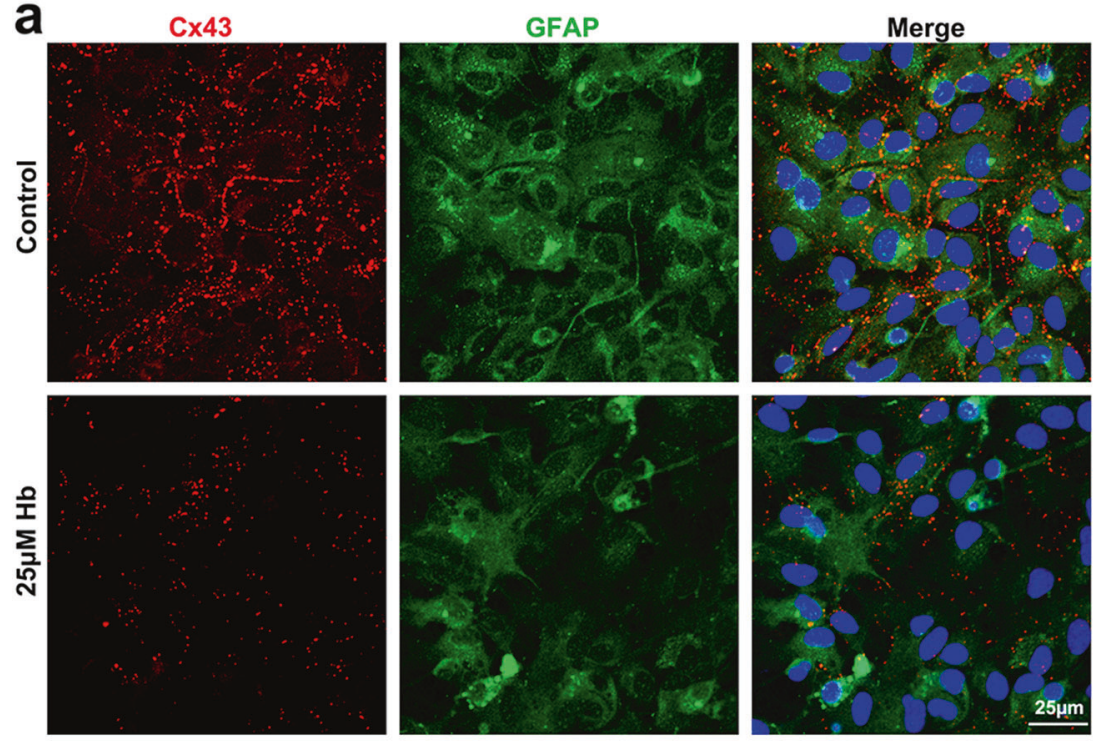

b

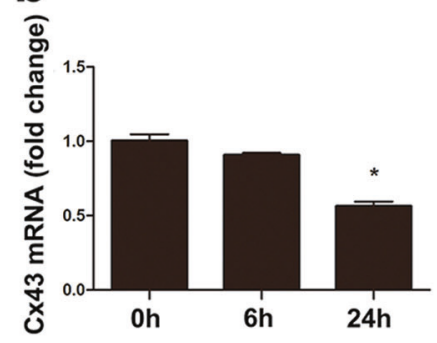

C

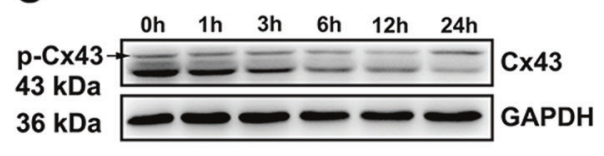

e

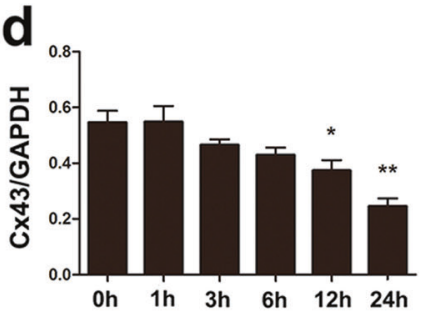

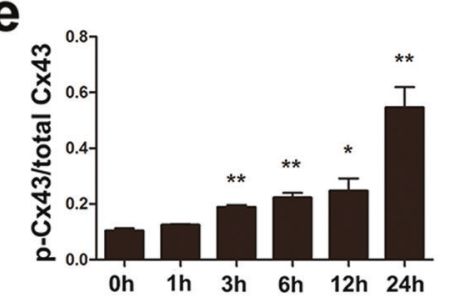

To examine its involvement following $\mathrm{ICH}$, we first traced YAP expression by parallel immunofluorescence staining and Western blotting analysis. These studies showed that YAP translocated to the nucleus upon $\mathrm{Hb}$ stimulation (Fig. 5a), an effect likely the result of reduced p-YAP/YAP after $\mathrm{Hb}$ treatment $(p<0.05)$ (Fig. 5b-d). Protein fractionation studies (Fig. 5e, f) corroborated the increase in nucleus/cytoplasmic YAP in $\mathrm{Hb}$ treated astrocytes than control $(p<0.05)$. The results indicated that Hb-treatment promoted YAP nuclear translocation.

To validate whether YAP nuclear translocation can also be observed in vivo, we performed immunohistochemistry staining for YAP in the ICH and Hb-injection models. These studies (Fig. 5g) showed clear YAP-positive nuclei in the peri-lesion area when compared with control brain.

\section{YAP inhibitor counteracted $\mathrm{Hb}$-induced proliferation and AMT}

YAP is transcriptionally active when it translocates to the nucleus and forms complexes with transcription factors (TEAD, FoxO1, Smad1, etc.). Verteporfin (VP), which breaks the association between YAP and TEAD, is widely accepted as a YAP inhibitor [41]. Consistent with this model, we noted that the proportion of Ki67-positive cells decreased dose-dependently after VP treatment $(p<0.05)$ (Fig. 6a, b). Additionally, Hb-induced switching from epithelial to mesenchymal marker expression was also reversed following VP treatment $(p<0.05)$ (Fig. 6c, d). These results supported a role for YAP signaling in Hb-induced proliferation and AMT in astrocytes. 

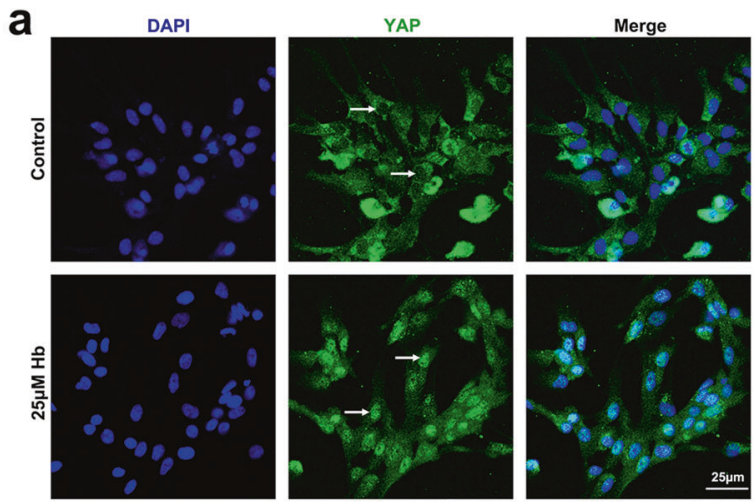

b

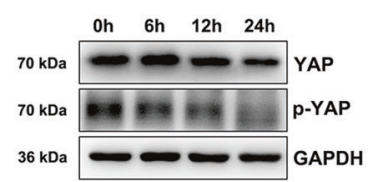

C

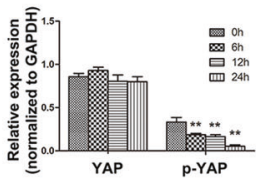

d
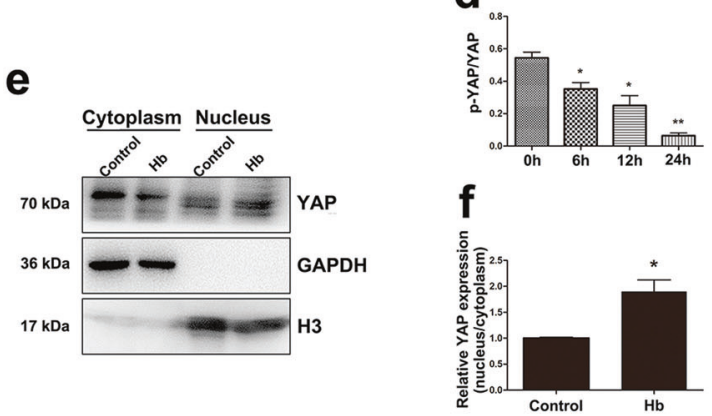

g
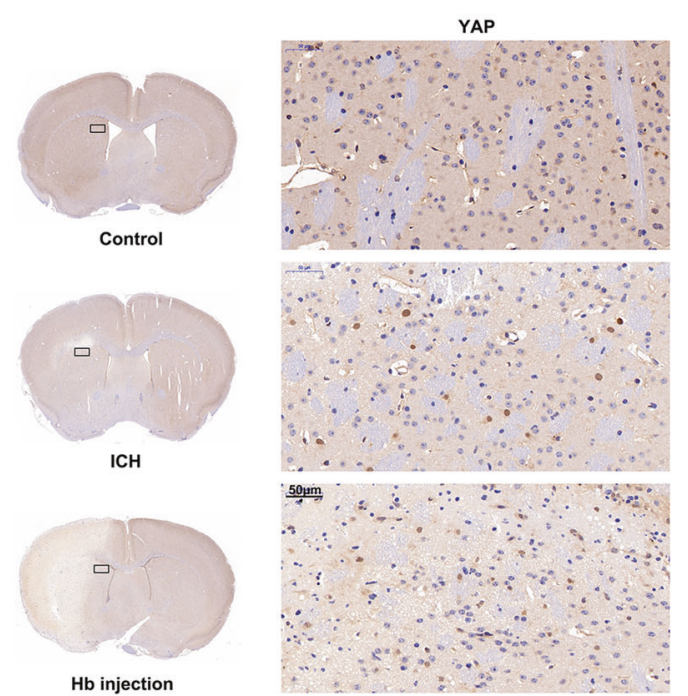

$\mathrm{Hb}$ injection

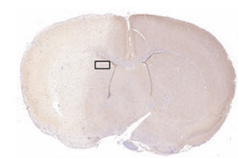

\section{Knocking down Cx43 triggered YAP nuclear translocation}

We next turned our attention to determine whether $\mathrm{Cx} 43$ downregulation and YAP nuclear translocation were intimately linked. To verify that YAP nuclear translocation was
Fig. $5 \mathrm{Hb}$ treatment induced YAP nuclear translocation in astrocytes. a Immunofluorescence staining of astrocytes with YAP (green) antibody. The cell nuclei were counterstained with DAPI (blue). The arrows indicate the differential localization of immunopositive products in the nuclei. $\mathrm{Hb}$ treatment promoted YAP nuclear translocation. Bar $=25 \mu \mathrm{m}$. b Western blotting analysis of YAP and p-YAP expression in astrocytes treated with $25 \mu \mathrm{M} \mathrm{Hb}$ for $0,6,12$, or $24 \mathrm{~h}$. $\mathbf{c , d}$ The results of densitometric analysis of YAP and p-YAP and pYAP/YAP were plotted as mean \pm SEM of three independent experiments. p-YAP and p-YAP/YAP expression was decreased upon $\mathrm{Hb}$ exposure. ${ }^{*} p<0.05,{ }^{* *} p<0.01$ compared with control $(0 \mathrm{~h})$. e Western blotting analysis of cytoplasmic and nucleus extraction samples from control and $25 \mu \mathrm{M} \mathrm{Hb}$ treated astrocytes with YAP antibody. GAPDH and H3 were used as loading control for cytoplasmic and nucleus protein, respectively. $\mathbf{f}$ The histogram showing the results of densitometric analysis of nucleus/cytoplasmic YAP expression in control and $25 \mu \mathrm{M} \mathrm{Hb}$ treated astrocytes. The results were normalized to control. $\mathrm{Hb}$ treatment significantly increased YAP nuclear expression. ${ }^{*} p<0.05,{ }^{* *} p<0.01$ compared with control. g Immunohistochemistry staining of YAP in control brain and the peri-lesion area of ICH model and Hb-injection model. Bar $=50 \mu \mathrm{m}$

triggered by $\mathrm{Cx} 43$ downregulation, we conducted RNA interference experiment. As indicated (Fig. 7a, b), over $80 \%$ of $\mathrm{Cx} 43$ was suppressed by si-Cx43 $(p<0.01)$. Immunofluorescence staining showed that YAP immunopositive products were mainly located in the cytoplasm in control and si-NC treated astrocytes. In contrast, YAP nuclear translocation was readily evident in si-Cx43 transfected astrocytes as shown by IF (Fig. 7c) and Western blotting analysis (Fig. 7d, e). These results indicated that $\mathrm{Cx} 43$ downregulation is an upstream event of YAP nuclear translocation.

\section{CX43 and YAP are physically associated}

It has been reported that cell-cell contact regulates the Hippo pathway by sequestrating YAP to adherens or junctions and suppressing its transcriptional activity [33]. Cx43-based gap junctions form when astrocytes make contact. To determine whether Cx43 and YAP are physically associated, we performed immunofluorescence staining and co-immunoprecipitation. As shown (Fig. 8a), Cx43 and YAP co-localize at gap junctional plaques $(r=0.229)$ where astrocytes make contact. Strikingly, following $\mathrm{Hb}$ treatment, the Cx43-YAP complex dissociates $(r=0.071)$, leading to YAP nuclear translocation. To quantify these events in vivo, we performed $\mathrm{Cx} 43$ and YAP immunofluorescence double staining on brain tissue slices (Fig. 8b), the Pearson's coefficients are $(r=0.142)$ in control and $(r=0.082)$ in ICH group. The immunofluorescence double staining was also performed in $\mathrm{Hb}$-injection model (Figure S3), the Pearson's coefficients are $(r=0.147)$ in control and $(r=0.060)$ in Hb-injection group. By YAP immunoprecipitation, $\mathrm{Cx} 43$ was detected in the precipitated complex (Fig. 8c). This finding was further verified by 
a
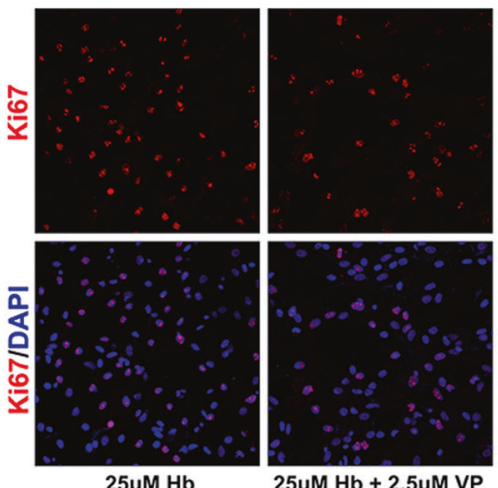

$25 \mu \mathrm{M} \mathrm{Hb}+2.5 \mu \mathrm{M} \mathrm{VP}$
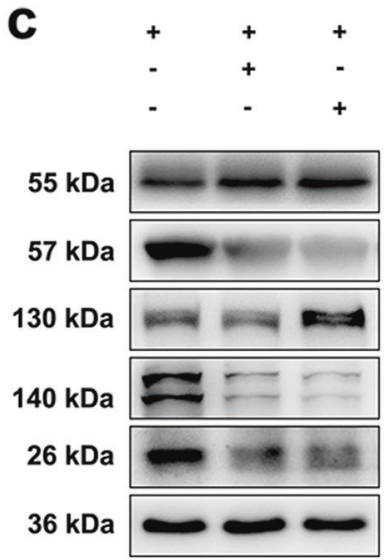

GFAP

Vimentin

E-Cadherin

$\mathrm{N}-$ Cadherin

SLUG

GAPDH b
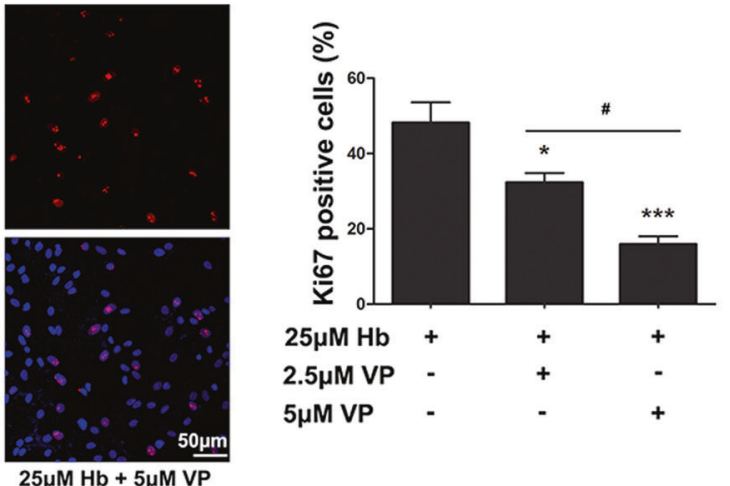

d

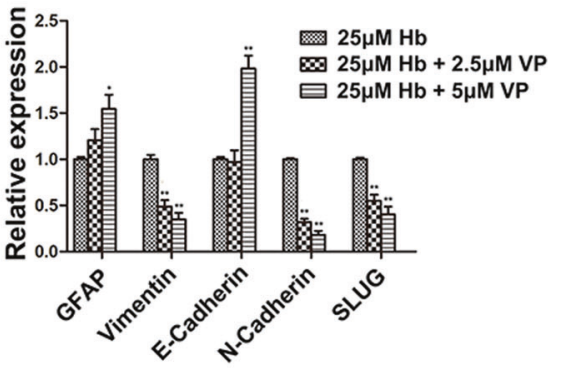

compared with $25 \mu \mathrm{M} \mathrm{Hb}+2.5 \mu \mathrm{M}$ VP treated group. c Western blotting analysis of GFAP, Vimentin, E-Cadherin, N-Cadherin, and SLUG expression in astrocytes treated with $25 \mu \mathrm{M} \mathrm{Hb}, 25 \mu \mathrm{M} \mathrm{Hb}+$ $2.5 \mu \mathrm{M} \mathrm{VP}, 25 \mu \mathrm{M} \mathrm{Hb}+5 \mu \mathrm{M} \mathrm{VP}$. The relative expression of the proteins was normalized to $25 \mu \mathrm{M} \mathrm{Hb}$ treated sample. $\mathbf{d}$ The results of densitometric analysis of the bands were plotted as mean \pm SEM of three independent experiments. VP partially reversed Hb-induced AMT. $* p<0.05, * * p<0.01$ compared with $25 \mu \mathrm{M}$ Hb treated group

CNS. Nevertheless, the molecular mechanism of astrocyte activation remains to be elucidated.

In our in vivo study, we observed increased Vimentin expression following $\mathrm{ICH}$, which was in line with the literature [37]. However, we also noticed a temporal and spatial difference in GFAP and Vimentin expression. With downregulation of GFAP, we noted upregulation of Vimentin at $12 \mathrm{~h}$ post-ICH. This was reminiscent of the switch that occurs in radial glia and immature astrocytes where Vimentin is progressively replaced by GFAP with differentiation into mature astrocytes [38]. This allowed us to propose that astrocytes may undergo de-differentiation following ICH. The de-differentiation of astrocytes is also reported in low temperature [42] or growth factor [16] treated astrocytes, it may be related to the cell proliferation. Vimentin expression reaches its peak earlier than GFAP. At 7d post-ICH, GFAP had the highest expression, whereas Vimentin expression level returned to resting state. We 
a

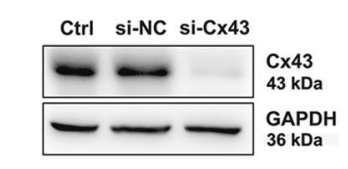

C
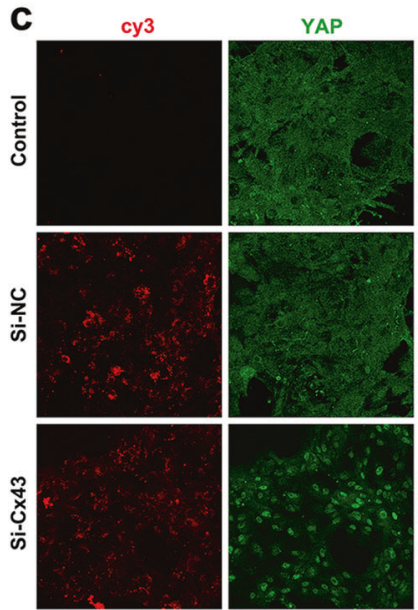

d

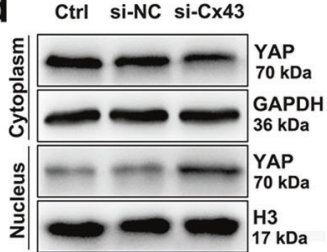

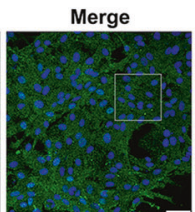

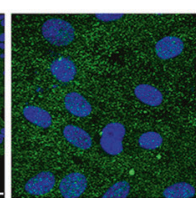

b
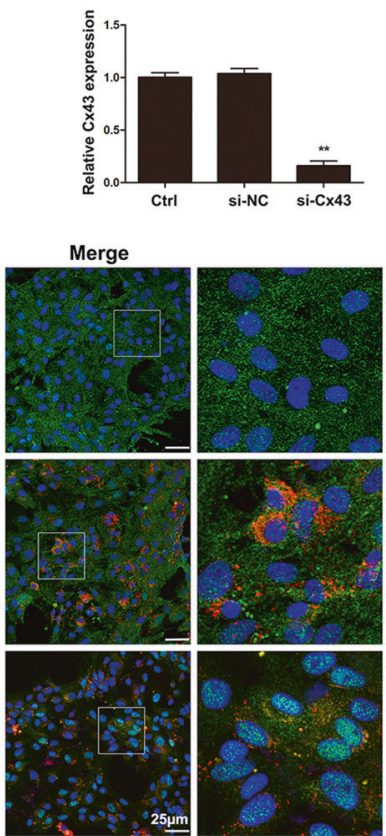

e

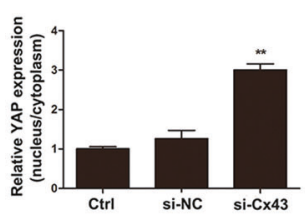

Fig. 7 Knocking down $\mathrm{Cx} 43$ triggered YAP nuclear translocation. a Western blotting analysis of $\mathrm{Cx} 43$ expression in control, si-NC, si$\mathrm{Cx} 43$ treated astrocytes. $\mathbf{b}$ The results of densitometric analysis of the bands were plotted as mean \pm SEM of three independent experiments. Over $80 \%$ of $\mathrm{Cx} 43$ expression was suppressed by si-Cx43. ** $p<0.01$ compared with control. $\mathbf{c}$ Immunofluorescence staining of control, si$\mathrm{NC}$, si-Cx43 treated astrocytes with YAP (green) antibody. The siRNA was labeled with fluorophore Cy3 (red) to show the transfected cells. The cell nuclei were counterstained with DAPI (blue). Bar $=25$ $\mu \mathrm{m}$. d Western blotting analysis of cytoplasmic and nucleus extraction samples from control, si-NC, si-Cx43 treated astrocytes with YAP antibody. GAPDH and $\mathrm{H} 3$ were used as loading control for cytoplasmic and nucleus protein, respectively. e The histogram showing the results of densitometric analysis of nucleus/cytoplasmic YAP expression in control, si-NC, si-Cx43 treated astrocytes. The results were normalized to control. si-Cx43 significantly increased YAP nuclear expression. $* * p<0.01$ compared with control

propose that reactive astrocytes with intensive GFAP expression may differentiate from previously Vimentinpositive mesenchymal-like immature astrocytes.

Our in vitro studies showed that $\mathrm{Hb}$ induced astrocyte activation, which was characterized by cell proliferation and upregulation of inflammatory cytokines. Our findings are consistent with Gram and colleagues' work on preterm intraventricular hemorrhage, which revealed that $\mathrm{Hb}$ induced IL- $1 \beta$ and TNF- $\alpha$ expression in primary rabbit pup astrocyte cultures by activating toll-like receptors [43].

Our in vitro studies showed that GFAP and E-cadherin were downregulated but Vimentin, N-Cadherin and SLUG were upregulated in cultured astrocytes upon $\mathrm{Hb}$ stimulation. These findings are consistent with our proposed AMT model. Our finding is distinct from most reported in vivo findings that intensified GFAP staining is the hallmark of reactive astrogliosis. We consider the reasons for the difference to be as follows: (1) Reactive astrogliosis characterized by intensive GFAP staining may be a late event of astrocyte activation as it is detectable 1, 3, 7, and 14 days post-ICH. In contrast, AMT is an early event detectable within the first $12 \mathrm{~h}$ post-ICH. (2) Purified astrocyte cultures are distinct from astrocytes in vivo. GFAP is readily detectable in cultured astrocytes, but is often not detectable in healthy brain tissue and tissue remote from the site of injury [8]. (3) Interactions between multiple cell populations in vivo can be difficult to detect in purified astrocyte cultures in vitro. (4) Although the increased expression of both GFAP and Vimentin was observed in several pathological models [44, 45], the temporal and spatial expression was not studied in detail.

It has been reported that radial glia and immature astrocytes express mainly Vimentin. Radial glia are derived from neuroepithelial progenitors. They share the elongated bipolar appearance with their ancestors, but express astroglial markers [46]. Radial glial cells represent the major neural progenitors and serve as the scaffold for neuron migration in the developing CNS [47]. It is reported that adult neural stem cells in the subventricular zone are derived from embryonic radial glia [48]. While radial glia are rare in the adult CNS, Müller glia are specialized radial glial cells in the adult retina that can de-differentiate into readily dividing neural progenitors in response to injury [49].

Using a selective model of $\mathrm{Hb}$ administration, we noted that this globin can induce astrocytes to express high levels of Vimentin comparable to those in immature astrocytes and radial glia [38]. From the perspective of genesis and the morphology, $\mathrm{Hb}$-induced Vimentin-positive astrocytes are not the typical radial glia, but they share some commonality. We propose that AMT may be a manifestation of $\mathrm{Hb}$ induced de-differentiation of astrocyte, which can be considered as partial reversion from the terminally differentiated cells. It is reminiscent of the partial dedifferentiation that astrocytes exhibit upon growth-factorstimulation [16]. Proliferation of astrocytes is a prerequisite for glial scar formation [50]. Thus we propose that $\mathrm{Hb}$ induced de-differentiation can prepare astrocytes for proliferation. Following this, the newly proliferated cells can undergo hypertrophy and get their processes overlapped and intertwined extensively to form the compact border which is referred to as a glial scar.

Derived from neuroectoderm, astrocytes are considered to be specialized epithelial cells in the CNS. Our proposed 

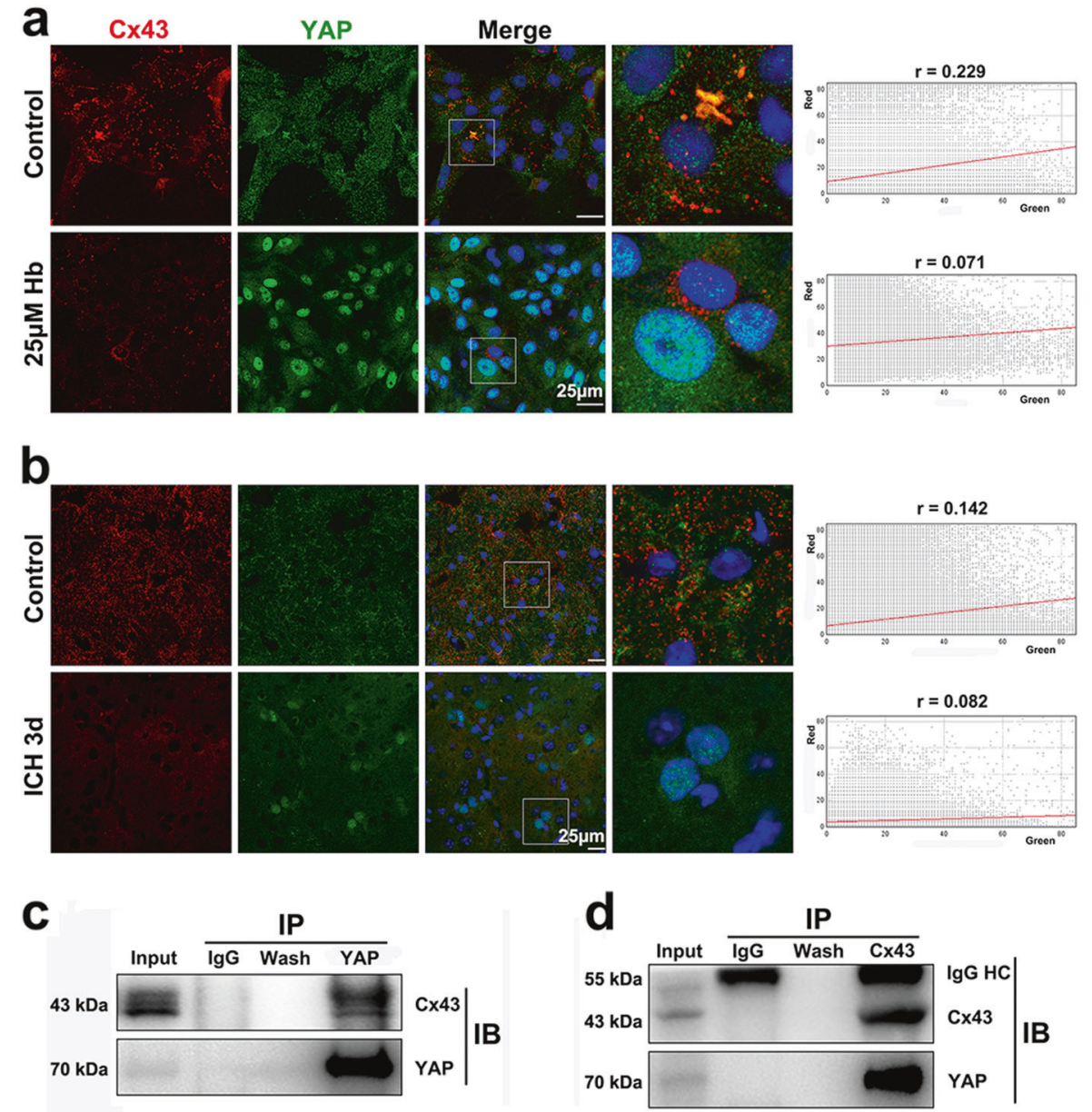

Fig. $8 \mathrm{Cx} 43$ and YAP were co-localized and physically associated. a Immunofluorescence double staining for $\mathrm{Cx} 43$ (red) and YAP (green) in astrocytes treated with or without $25 \mu \mathrm{M} \mathrm{Hb}$. The cell nuclei were counterstained with DAPI (blue). Co-localization of the two protein was observed at the sites where cell-cell contact in untreated astrocytes. After $\mathrm{Hb}$ stimulation, $\mathrm{Cx} 43$ disappeared from the cell membrane and YAP accumulated in the nuclei. In the scatter plot, the symbol $r$ represents the Pearson's correlation coefficient. Bar $=25 \mu \mathrm{m}$. b Immunofluorescence double staining for $\mathrm{Cx} 43$ (red) and YAP (green) in control and ICH brain tissue slices. The cell nuclei were counterstained with DAPI (blue). Co-localization of the two proteins were

AMT model is analogous to the well-studied EMT. EMT was first described in embryonic development and is characterized by loss of epithelial cell polarity and cell-cell adhesion, yielding migratory properties [51]. EMT is widely involved in tissue repair, regeneration, and fibrosis [52]. $\mathrm{ICH}$ is a severe injury to the CNS where astrocytes undergo reactive astrogliosis analogous to fibrosis. We propose that the de-differentiation and AMT of astrocytes contribute to reactive astrogliosis by facilitating the proliferation and migration of reactive astrocytes.

Cx43-based intercellular gap junctions are the predominant cell adhesions in homeostatic astrocytes. The half-life of $\mathrm{Cx} 43$ in cultured cells is less than $5 \mathrm{~h}$. Its

also observed in the control brain. $\mathrm{Cx} 43$ and YAP dissociated and YAP appeared in some of the nuclei in the peri-lesion area of ICH brain. In the scatter plot, the symbol $r$ represents the Pearson's correlation coefficient. Bar $=25 \mu \mathrm{m}$. $\mathbf{c}$ Western blotting analysis of YAP immunoprecipitated samples using rabbit anti-Cx43 polyclonal antibody. Cx43 was detected in the immunoprecipitated complex. d Western blotting analysis of $\mathrm{Cx} 43$ immunoprecipitated samples using rabbit anti-YAP polyclonal antibody. YAP was detected in the immunoprecipitated complex. IgG $\mathrm{HC}$ means the heavy chain of the immunoglobin

degradation is quickly modulated by phosphorylation [23]. It is reported that pro-inflammatory cytokines promote Cx43 degradation via the ubiquitin proteasome system [53]. Glioma, which is characterized by the uncontrolled proliferation of poorly differentiated astrocytes, is an example of cell destabilization. It is reported that downregulation of $\mathrm{Cx} 43$ is related to the malignant transformation of glioma $[24,25]$. In our research, Hb-induced astrocytes activation disrupted homeostasis. We found that $\mathrm{Cx} 43$ was downregulated with increased $\mathrm{p}-\mathrm{Cx} 43 / \mathrm{Cx} 43$ upon $\mathrm{Hb}$ stimulation. Cx43 phosphorylation leads to its conformational changes, channel closure, and degradation [23]. Based on Zhang's work [53], we propose that downregulation of 
$\mathrm{Cx} 43$ in Hb-activated astrocytes is the result of inflammatory cytokines produced, but the mechanism needs further exploration. Because $\mathrm{Cx} 43$ was phosphorylated and downregulated in mitotic cells [23], we propose that downregulation of $\mathrm{Cx} 43$ may facilitate astrocyte detachment and proliferation.

The Hippo pathway is well-accepted as an important regulator of tissue homeostasis. Dysregulation of the Hippo pathway is highly relevant to tumor development [26]. As a key effector of the Hippo pathway, YAP is transcriptionally active when it translocates to the nucleus to form complexes with transcription factors. In our study, YAP nuclear translocation was observed in $\mathrm{Hb}$-treated astrocytes as well as in the ICH brain. In addition, we found $\mathrm{Hb}$ treatment endowed astrocytes with increased proliferation and astroglial-mesenchymal phenotype switching; an effect arrested through co-administration of a YAP inhibitor. Our findings indicate that the astrocytic response to $\mathrm{Hb}$ exposure was at least partially YAP dependent. However, Huang and colleagues reported that YAP knockout mice developed reactive astrogliosis spontaneously [36], indicating that YAP may be a negative regulator of reactive astrogliosis. Their conclusion is different from ours possibly due to the highly distinct models of brain injury compared with genetic ablation.

Further we noted that knock down of Cx43 triggers YAP nuclear translocation. It is reported that cell adhesion and tight junction related proteins such as Angiomotin (AMOT), protein tyrosine phosphatase non-receptor type 14 (PTPN14), and $\alpha$-catenin contribute to sequestering YAP at cell junctions [26]. We conducted immunofluorescence double staining and co-immunoprecipitation studies to examine the physical association between $\mathrm{Cx} 43$ and YAP. The results confirmed our hypothesis. Whether they are binding directly or mediated by other proteins remains to be determined.

Taken together, we propose that YAP is sequestered to astroglial gap junctions through direct or indirect interactions with $\mathrm{Cx} 43$. Upon $\mathrm{Hb}$ stimulation, $\mathrm{Cx} 43$ undergoes phosphorylation and degradation. This liberates YAP and allows its nuclear translocation where it can transcriptionally activate and promote the expression of genes involved in cell proliferation and AMT (Figure S4).

To our knowledge, this is the first study to report astroglial-mesenchymal phenotype switching during $\mathrm{Hb}$ induced astrocyte activation. Activated astrocytes acquire enhanced proliferation and mesenchymal features, which may contribute to reactive astrogliosis. $\mathrm{Cx} 43$ downregulation-facilitated YAP nuclear translocation is a novel mechanism involved in this process. Cx43-YAP interaction may represent a potentially important therapeutic target in the management of $\mathrm{ICH}$.

\section{Materials and methods}

\section{ICH mouse model}

All experimental procedures were approved by the Shanghai Jiao Tong University experimental ethics committee. 10-week-old male mice were anesthetized with a single intraperitoneal dose of ketamine $(100 \mathrm{mg} / \mathrm{kg})$ and xylazine $(10 \mathrm{mg} / \mathrm{kg})$. The mice were secured in a stereotactic frame (RWD Life Science co., Shenzhen, China) and subjected to ICH using collagenase IV (Sigma-Aldrich, MO, USA) as previously described [54] with modification. A $1 \mathrm{~mm}$ diameter burr hole was drilled $2.2 \mathrm{~mm}$ lateral to the midline and $0.5 \mathrm{~mm}$ anterior to bregma. The needle was advanced $3 \mathrm{~mm}$ into the right striatum. A total of $0.075 \mathrm{U}$ collagenase IV dissolved in $0.4 \mu \mathrm{L}$ phosphate buffer (PBS) was injected over $90 \mathrm{~s}$ using a microinfusion pump (WPI, Sarasota, FL). The needle was left in place for $5 \mathrm{~min}$ to avoid the reflux. After withdrawal of the needle, the burr hole was sealed with bone wax and the scalp was sutured. The animals were allowed to recover on a $37^{\circ} \mathrm{C}$ heating pad after operation.

After being deeply anesthetized, mice were perfused with saline followed by fixation with $4 \%$ paraformaldehyde (PFA) in $0.1 \mathrm{~mol} / \mathrm{L}$ PBS at $12 \mathrm{~h}, 1 \mathrm{~d}, 3 \mathrm{~d}, 7 \mathrm{~d}$, and $14 \mathrm{~d}$ postoperation. Brains were dehydrated in $30 \%$ sucrose after an overnight postfixation in 4\% PFA. Brain cryosections $(20 \mu \mathrm{m})$ were prepared and then subjected to immunofluorescence staining. For Western blotting analysis, the mice were killed at $12 \mathrm{~h}, 1 \mathrm{~d}, 3 \mathrm{~d}, 7 \mathrm{~d}$, and $14 \mathrm{~d}$ postoperation. The peri-lesion area was collected and subjected to lysate preparation and Western blotting analysis.

\section{Astrocyte cultures}

Primary astrocyte cultures were prepared from newborn Sprague Dawley rats (SLAC, Shanghai, China), as described previously [55]. In brief, after brain cortices were dissected, the meninges, blood vessels, and hippocampus were removed under a microscope. The remaining cortical tissue was trypsinized for $10 \mathrm{~min}$ at $37^{\circ} \mathrm{C}$, and dissociated by gentle trituration. The cell pellet was collected by centrifugation, resuspended in culture medium (90\% Dulbecco modified Eagle medium (DMEM), 10\% fetal bovine serum, $100 \mathrm{U} / \mathrm{mL}$ penicillin $\mathrm{G}$, and $100 \mathrm{mg} / \mathrm{mL}$ streptomycin sulfate) and plated onto poly-D-lysine-coated $75 \mathrm{~cm}^{2}$ flasks, maintained at $37{ }^{\circ} \mathrm{C}$ in $95 \%$ humidity with $5 \% \mathrm{CO}_{2}$. The culture medium was refreshed every 4 days. When the cells reached confluence, microglia and oligodendrocyte progenitors were removed by shaking the flask at $220 \mathrm{rpm}$ for $1 \mathrm{~h}$ at $37^{\circ} \mathrm{C}$. The pure secondary astrocytes (over 99\%), as verified by immunofluorescence staining (GFAP for astrocytes, Iba-1 for microglia), were used in the experiments. 


\section{RNA interference experiment}

Cy3 labeled negative control siRNA (si-NC) and Cx43 siRNA (si-Cx43) were purchased from Genepharm (Shanghai, China). The sequences are as follows: si-NC sense: 5'-UUCUCCGAACGUGUCACGUTT-3', si-NC antisense: 5'-ACGUGACACGUUCGGAGAATT-3', siCx43 sense: $5^{\prime}$-ACAUCAUUGAGCUCUUCUATT- ${ }^{\prime}$, siCX43 antisense: $5^{\prime}$-UAGAAGAGCUCAAUGAUGUTT- $3^{\prime}$. Cells were seeded at a density of $5 \times 10^{5}$ cells per dish in 6 $\mathrm{cm}$-dish or $2 \times 10^{4}$ cells per well in 24 -well plate, for Western blotting analysis or immunofluorescence staining, respectively. Twenty-four hours later, the cells were transfected with the siRNAs with Lipoectamine 2000 according to the manufacturer's instructions. The medium was replaced $24 \mathrm{~h}$ later to remove the excessive transfection complex. $72 \mathrm{~h}$ after transfection, the cells were harvested and used for subsequent analysis.

\section{Cell viability assay}

Cell viability was determined using the Cell Counting Kit-8 (CCK-8) (Beyotime, Jiangsu, China) assay following the manufacturer's instructions. Briefly, cells were plated into 96-well plates at a density of $1 \times 10^{4}$ cells per well in 100 $\mu \mathrm{L}$ culture medium. Twenty-four hours later, the cells were exposed to $0,10,20,30$, or $50 \mu \mathrm{M} \mathrm{Hb}$ (Beyotime, Jiangsu, China) for $24 \mathrm{~h}$, then $10 \mu \mathrm{L}$ CCK- 8 solution was added into each well. After incubating for $4 \mathrm{~h}$ at $37^{\circ} \mathrm{C}$ with $5 \% \mathrm{CO}_{2}$, absorbance at $450 \mathrm{~nm}$ was measured using a microplate reader (BioTek, VT, USA).

\section{Cell proliferation assessment}

Astrocytes were seeded onto the $15 \mathrm{~mm}$-diameter coverslips at a density of $5 \times 10^{4}$, and $24 \mathrm{~h}$ later, the cells were treated with or without $25 \mu \mathrm{M} \mathrm{Hb}$ for $24 \mathrm{~h}$. In additional experiments, 2.5 or $5 \mu \mathrm{M}$ verteporfin (VP) (Cat\# SML0534, Sigma-Aldrich, MO, USA) were administered with $\mathrm{Hb}$. Then the cells were fixed in 4\% PFA for $10 \mathrm{~min}$ and then subjected to Ki67 immunofluorescence staining. Fluorescence images were acquired using a confocal laser-scanning microscope (Leica, Solms, Germany). The average percentage of Ki67-positive cells was calculated from 10 random visual fields from each sample.

\section{Total RNA extraction and quantitative real-time PCR analysis}

Astrocytes were plated into 6-well plates at a density of $5 \times$ $10^{5}$ cells per well, after treatment with or without $25 \mu \mathrm{M} \mathrm{Hb}$ for 6 or $24 \mathrm{~h}$, total RNA was extracted using TRIzol reagent (Invitrogen, CA, USA). Reverse transcription was performed using PrimeScript ${ }^{\mathrm{TM}} \mathrm{RT}$ reagent kit with gDNA Eraser (Takara, Dalian, China). IL-1 $\beta$, IL-6, TNF- $\alpha$, and Cx43 mRNA expression was quantified using SYBR $^{\circledR}$ Premix Ex Taq ${ }^{\mathrm{TM}}$ (Tli RNaseH Plus) (Takara, Dalian, China). The oligonucleotide primers used were as follows:

\begin{tabular}{lll} 
Gene name & Forward $\left(5^{\prime}-3^{\prime}\right)$ & Reverse $\left(5^{\prime}-3^{\prime}\right)$ \\
IL-1 $\beta$ & GTCTGACCCA & GCCACAGGGA \\
& TGTGAGCTG & TTTTGTCGTT \\
IL-6 & TACCCCAACT & GGTTTGCCGA \\
& TCCAATGCTC & GTAGACCTCA \\
TNF- $\alpha$ & TGATCGGTCC & TGCTTGGTGG \\
& CAACAAGGA & TTTGCTACGA \\
Connexin43 & CTCGCCTATG & TTTGCTCTGC \\
& TCTCCTCCTG & GCTGTAGTTC \\
GAPDH & GATGGTGAAG & TGAACTTGCC \\
& GTCGGTGTGA & GTGGGTAGAG \\
\hline
\end{tabular}

The data were normalized to the internal reference glyceraldehyde 3-phosphate dehydrogenase (GAPDH). The fold-change values were calculated by normalizing to control samples. PCR amplification was performed for 40 cycles, data were collected using SDS software (Applied Biosystems, CA, USA).

\section{Immunofluorescence staining}

The brain cryosections and the coverslips seeded with astrocytes were immunofluorescence stained following the routine procedure. The primary antibodies used were as follow: rabbit anti-YAP polyclonal antibody (1:100, Cat\# sc-15407, Santa Cruz Biotechnology, CA, USA), rabbit anti-Connexin43 polyclonal antibody (1:300, Cat\# SAB4501175, Sigma-Aldrich, MO, USA), goat anti-GFAP polyclonal antibody (1:100, Cat\# sc-6170, Santa Cruz Biotechnology, CA, USA), mouse anti-Vimentin monoclonal antibody (1:300, Cat\# MAB3400, Millipore, MA, USA), rabbit anti-Ki67 polyclonal antibody (1:500, Cat\# ab15580, Abcam, UK), rabbit anti-Adolase-C polyclonal antibody (1:200, Cat\# ab200049, Abcam, UK), rabbit antiIba-1 polyclonal antibody (1:200, Cat\# 019-19741, WAKO, Japan), rabbit anti-NeuN polyclonal antibody (1:500, Cat\# ab177487, Abcam, UK), rat anti-E-Cadherin polyclonal antibody (1:200, Cat\# ab11512, Abcam, UK), rabbit anti-NCadherin polyclonal antibody (1:200, Cat\# ab76057, Abcam, UK). The secondary antibodies used (1:300, Invitrogen, CA, USA): Alexa Fluor 488 donkey anti-rabbit IgG (Cat\# A21206), Alexa Fluor 594 donkey anti-rabbit IgG (Cat\# A21207), Alexa Fluor 594 donkey anti-rat IgG (Cat\# A21209), Alexa Fluor 647 donkey anti-rabbit IgG (Cat\# A31573), Alexa Fluor 647 donkey anti-mouse IgG (Cat\# 
A31571), Alexa Fluor 488 donkey anti-goat IgG (Cat\# A11055), and Alexa Fluor 594 donkey anti-mouse $\operatorname{IgG}$ (Cat\# A21203). Nuclei were counterstained with DAPI (1:5000, Invitrogen, CA, USA). Fluorescence images were captured using a confocal laser-scanning microscope (Leica, Solms, Germany). The Image J Just Another Colocalization Plugin (JACoP) was applied for quantitative colocalization analyses [56].

\section{Preparation of cell extracts and Western blotting analysis}

Astrocytes were plated into six-well plates at a density of $5 \times 10^{5}$ cells per well, and treated with $0,10,15$, and $25 \mu \mathrm{M}$ $\mathrm{Hb}$ for $24 \mathrm{~h}$, or with $25 \mu \mathrm{M} \mathrm{Hb}$ for $0,6,12$, and $24 \mathrm{~h}$. In additional experiments, cells treated with $25 \mu \mathrm{M} \mathrm{Hb}$ were simultaneously treated with or without 2.5 or $5 \mu \mathrm{M}$ VP. The cells were collected and whole cell lysates were prepared using RIPA lysis buffer (Millipore, MA, USA) supplemented with protease inhibitor cocktail (Roche, Basel, Swiss). Nucleus and cytoplasmic protein was extracted from cell cultures using a nuclear and cytoplasmic protein extraction kit (Beyotime, Jiangsu, China). Protein concentrations were determined with the Pierce ${ }^{\mathrm{TM}} \mathrm{BCA}$ Protein Assay Kit (ThermoFisher Scientific, MA, USA). Equal amounts of protein $(30 \mu \mathrm{g})$ were separated by standard sodium dodecyl sulfate polyacrylamide gel electrophoresis and then electrotransferred onto PVDF membranes. The blots were blocked with 5\% non-fat milk and incubated overnight at $4{ }^{\circ} \mathrm{C}$ with the appropriate primary antibodies: rabbit anti-Connexin 43 polyclonal antibody (1:1000, Cat\# SAB4501175, Sigma, MO, USA), mouse anti-GFAP monoclonal antibody (1:3000, Cat\# MAB3402, Millipore, MA, USA), mouse anti-Vimentin monoclonal antibody (1:3000, Cat\# MAB3400, Millipore, MA, USA), rabbit anti-E-cadherin polyclonal antibody (1:1000, Cat\# YT1454, Immunoway, TX, USA), rabbit anti-N-cadherin polyclonal antibody (1:1000, Cat\# YT2988, Immunoway, TX, USA), rabbit anti-SLUG polyclonal antibody (1:1000, Cat\# YM3371, Immunoway, TX, USA), rabbit anti-YAP monoclonal antibody (1:1000, Cat\# 14074, Cell Signaling Technology, MA, USA), rabbit anti-Phospho-YAP(S127) monoclonal antibody (1:1000, Cat\# 13008, Cell Signaling Technology, MA, USA), rabbit anti-histone-H3 monoclonal antibody (1:1000, Cat\# 4499, Cell Signaling Technology, MA, USA), and rabbit anti-GAPDH monoclonal antibody (1:2000, Cat\# 5174, Cell Signaling Technology, MA, USA). Subsequently, the membranes were washed thrice, then incubated with corresponding horseradish peroxidase conjugated secondary antibody for $1 \mathrm{~h}$ at room temperature. After washing, the membranes were incubated with ECL solution (ThermoFisher Scientific, MA, USA) for $3 \mathrm{~min}$. The signal was detected using the Tanon image system (Shanghai, China). Densitometric analysis of the bands was performed using ImageJ 1.6.0 (NIH, MD, USA).

\section{Co-immunoprecipitation of Cx43 and YAP}

Astrocyte cultures at $100 \%$ confluence were lysed with IP lysis buffer containing protease inhibitors (Beyotime, Jiangsu, China) and subsequently centrifuged at $12,000 \mathrm{~g}$ for $10 \mathrm{~min}$ at $4{ }^{\circ} \mathrm{C}$. The supernatant was used for coimmunoprecipitation. The protein concentration was estimated with Pierce ${ }^{\mathrm{TM}}$ BCA Protein Assay Kit (ThermoFisher Scientific, MA, USA). After preclearing with $20 \mu \mathrm{L}$ washed protein A/G agarose (Cat\# sc-2003, Santa Cruz Biotechnology, CA, USA), the lysate (1 mg protein) was incubated with $1 \mu \mathrm{g}$ mouse anti-Cx 43 monoclonal antibody (Cat\# C8093, Sigma, MO, USA), or normal mouse IgG (Cat\# sc-2025, Santa Cruz Biotechnology, CA, USA) for 6 $\mathrm{h}$ at $4{ }^{\circ} \mathrm{C}$. Then $20 \mu \mathrm{L}$ washed protein $\mathrm{A} / \mathrm{G}$ agarose was added to the complex, and rotated for $1 \mathrm{~h}$ at $4{ }^{\circ} \mathrm{C}$. The agarose was collected by centrifugation at $1000 \mathrm{~g}$ for $2 \mathrm{~min}$. After washing thrice with $1 \mathrm{~mL}$ IP lysis buffer, the protein bound to the agarose was eluted by boiling in $20 \mu \mathrm{L} 2 \times$ loading buffer and analyzed by Western blotting using rabbit anti-YAP monoclonal antibody (1:1000, Cat\# 14074, Cell Signaling Technology, CA, USA) to evaluate the association between $\mathrm{Cx} 43$ and YAP protein. Mouse antiYAP monoclonal antibody (Cat\# MAB8094, R\&D system, MN, USA) was used for additional immunoprecipitation and the precipitated complex was detected with rabbit antiConnexin 43 polyclonal antibody (1:1000, Cat\# SAB4501175, Sigma, MO, USA) for a further verification.

\section{Statistical analysis}

All data are presented as the mean \pm standard error of the mean (SEM) of at least three independent experiments. Statistical significance was evaluated using Student's $t$-test or One-way ANOVA followed by Post Hoc LeastSignificant Difference (LSD) tests. $p$-values $<0.05$ were considered to be significant. Statistical analysis was performed using SPSS 18.0 (IBM, NY, USA). Statistical charts were drawn using GraphPad Prism 5 (GraphPad Software, CA, USA).

Acknowledgements We sincerely thank Professor Shereen Ezzat from Princess Margaret Cancer Centre, University of Toronto for valuable suggestions and language polishing. We thank the other members of neuroscience and neuroengineering research center for technical support and helpful discussions. Financial support is from National Natural Science Foundation of China (Grant No. 81471176, 81671194, 81602180, 81601054, and 81771318) and SJTU MedicineEngineering Research Fund (Grant No. YG2014QN17 and YG2015QN40). 
Author contributions YY and LB designed the research, analyzed the results, and wrote the manuscript. YY and JR performed all molecular and cellular assays. YS prepared the ICH mouse model. YX collected the data and performed the statistical analysis. ZZ and AG provided useful suggestions on experiment design and reviewed the paper. BW, $\mathrm{ZZ}, \mathrm{ZC}$, and $\mathrm{ZX}$ assist with the IF, WB, IP, and cell culture. GY and QS assisted with reviewing and editing the paper. LB provided expertize and feedback.

\section{Compliance with ethical standards}

Conflict of interest The authors declare that they have no conflict of interest.

\section{References}

1. Mracsko E, Veltkamp R. Neuroinflammation after intracerebral hemorrhage. Front Cell Neurosci. 2014;8:388.

2. Aronowski J, Zhao X. Molecular pathophysiology of cerebral hemorrhage: secondary brain injury. Stroke. 2011;42:1781-6.

3. Zhou Y, Wang Y, Wang J, Anne Stetler R, Yang QW. Inflammation in intracerebral hemorrhage: from mechanisms to clinical translation. Prog Neurobiol. 2014;115:25-44.

4. Moeendarbary E, Weber IP, Sheridan GK, Koser DE, Soleman S, Haenzi B, et al. The soft mechanical signature of glial scars in the central nervous system. Nat Commun. 2017;8:14787.

5. Kielian T. Glial connexins and gap junctions in CNS inflammation and disease. J Neurochem. 2008;106:1000-16.

6. Li T, Giaume C, Xiao L. Connexins-mediated glia networking impacts myelination and remyelination in the central nervous system. Mol Neurobiol. 2014;49:1460-71.

7. Macco R, Pelizzoni I, Consonni A, Vitali I, Giacalone G, Martinelli Boneschi $\mathrm{F}$, et al. Astrocytes acquire resistance to irondependent oxidative stress upon proinflammatory activation. J Neuroinflamm. 2013;10:130.

8. Sofroniew MV, Vinters HV. Astrocytes: biology and pathology. Acta Neuropathol. 2010;119:7-35.

9. Jiang R, Diaz-Castro B, Looger LL, Khakh BS. Dysfunctional calcium and glutamate signaling in striatal astrocytes from Huntington's disease model mice. J Neurosci: Off J Soc Neurosci. 2016;36:3453-70.

10. Dooves S, Bugiani M, Postma NL, Polder E, Land N, Horan ST, et al. Astrocytes are central in the pathomechanisms of vanishing white matter. J Clin Invest. 2016;126:1512-24.

11. Capani F, Quarracino C, Caccuri R, Sica RE. Astrocytes as the main players in primary degenerative disorders of the human central nervous system. Front Aging Neurosci. 2016;8:45.

12. Almad AA, Doreswamy A, Gross SK, Richard JP, Huo Y, Haughey N, et al. Connexin 43 in astrocytes contributes to motor neuron toxicity in amyotrophic lateral sclerosis. Glia. 2016;64:1154-69.

13. Cekanaviciute E, Buckwalter MS. Astrocytes: integrative regulators of neuroinflammation in stroke and other neurological diseases. Neurother: J Am Soc Exp Neurother. 2016;13:685-701.

14. Anderson MA, Ao Y, Sofroniew MV. Heterogeneity of reactive astrocytes. Neurosci Lett. 2014;565:23-29.

15. Di Giovanni S, Movsesyan V, Ahmed F, Cernak I, Schinelli S, Stoica B, et al. Cell cycle inhibition provides neuroprotection and reduces glial proliferation and scar formation after traumatic brain injury. Proc Natl Acad Sci USA. 2005;102:8333-8.

16. Puschmann TB, Zanden C, Lebkuechner I, Philippot C, de Pablo Y, Liu J, et al. HB-EGF affects astrocyte morphology, proliferation, differentiation, and the expression of intermediate filament proteins. J Neurochem. 2014;128:878-89.
17. Schulz R, Gorge PM, Gorbe A, Ferdinandy P, Lampe PD, Leybaert L. Connexin 43 is an emerging therapeutic target in ischemia/reperfusion injury, cardioprotection and neuroprotection. Pharmacol Ther. 2015;153:90-106.

18. Westphalen K, Gusarova GA, Islam MN, Subramanian M, Cohen TS, Prince AS, et al. Sessile alveolar macrophages communicate with alveolar epithelium to modulate immunity. Nature. 2014;506:503-6.

19. Boulay AC, Cisternino S, Cohen-Salmon M. Immunoregulation at the gliovascular unit in the healthy brain: a focus on connexin 43 . Brain Behav Immun. 2016;56:1-9.

20. Boulay AC, Mazeraud A, Cisternino S, Saubamea B, Mailly P, Jourdren L, et al. Immune quiescence of the brain is set by astroglial connexin 43. J Neurosci: Off J Soc Neurosci. 2015;35:4427-39.

21. Le HT, Sin WC, Lozinsky S, Bechberger J, Vega JL, Guo XQ, et al. Gap junction intercellular communication mediated by connexin43 in astrocytes is essential for their resistance to oxidative stress. J Biol Chem. 2014;289:1345-54.

22. Zhou JJ, Cheng C, Qiu Z, Zhou WH, Cheng GQ. Decreased connexin 43 in astrocytes inhibits the neuroinflammatory reaction in an acute mouse model of neonatal sepsis. Neurosci Bull. 2015;31:763-8.

23. Solan JL, Lampe PD. Connexin 43 phosphorylation: structural changes and biological effects. Biochem J. 2009;419:261-72.

24. Sin WC, Aftab Q, Bechberger JF, Leung JH, Chen H, Naus CC. Astrocytes promote glioma invasion via the gap junction protein connexin43. Oncogene. 2016;35:1504-16.

25. Yu SC, Xiao HL, Jiang XF, Wang QL, Li Y, Yang XJ, et al. Connexin 43 reverses malignant phenotypes of glioma stem cells by modulating E-cadherin. Stem Cells. 2012;30:108-20.

26. Yu FX, Guan KL. The Hippo pathway: regulators and regulations. Genes \& Dev. 2013;27:355-71.

27. Lin AY, Pearson BJ. Planarian yorkie/YAP functions to integrate adult stem cell proliferation, organ homeostasis and maintenance of axial patterning. Development. 2014;141:1197-208.

28. Lian I, Kim J, Okazawa H, Zhao J, Zhao B, Yu J, et al. The role of YAP transcription coactivator in regulating stem cell self-renewal and differentiation. Genes Dev. 2010;24:1106-18.

29. Liu Y, Wang G, Yang Y, Mei Z, Liang Z, Cui A, et al. Increased TEAD4 expression and nuclear localization in colorectal cancer promote epithelial-mesenchymal transition and metastasis in a YAP-independent manner. Oncogene. 2016;35:2789-2800.

30. Song Y, Fu J, Zhou M, Xiao L, Feng X, Chen H, et al. Activated hippo/yes-associated protein pathway promotes cell proliferation and anti-apoptosis in endometrial stromal cells of endometriosis. $\mathrm{J}$ Clin Endocrinol Metab. 2016;101:1552-61.

31. Yu FX, Zhao B, Guan KL. Hippo pathway in organ size control, tissue homeostasis, and cancer. Cell. 2015;163:811-28.

32. Cox CM, Mandell EK, Stewart L, Lu R, Johnson DL, McCarter $\mathrm{SD}$, et al. Endosomal regulation of contact inhibition through the AMOT:YAP pathway. Mol Biol Cell. 2015;26:2673-84.

33. Zhao B, Wei X, Li W, Udan RS, Yang Q, Kim J, et al. Inactivation of YAP oncoprotein by the Hippo pathway is involved in cell contact inhibition and tissue growth control. Genes \& Dev. 2007;21:2747-61.

34. Huang Z, Xiong WC. Neogenin-YAP signaling in neocortical astrocytic differentiation. Neurogenesis. 2016;3:e1248735.

35. Huang Z, Sun D, Hu JX, Tang FL, Lee DH, Wang Y, et al. Neogenin promotes BMP2 activation of YAP and Smad1 and enhances astrocytic differentiation in developing mouse neocortex. J Neurosci: Off J Soc Neurosci. 2016;36:5833-49.

36. Huang Z, Wang Y, Hu G, Zhou J, Mei L, Xiong WC. YAP is a critical inducer of SOCS3, preventing reactive astrogliosis. Cereb Cortex. 2016;26:2299-310. 
37. Hol EM, Pekny M. Glial fibrillary acidic protein (GFAP) and the astrocyte intermediate filament system in diseases of the central nervous system. Curr Opin Cell Biol. 2015;32:121-30.

38. Bramanti V, Tomassoni D, Avitabile M, Amenta F, Avola R. Biomarkers of glial cell proliferation and differentiation in culture. Front Biosci. 2010;2:558-70.

39. Thompson RJ, Kynoch PA, Willson VJ. Cellular localization of aldolase C subunits in human brain. Brain Res. 1982;232:489-93.

40. Kalluri R, Weinberg RA. The basics of epithelial-mesenchymal transition. J Clin Invest. 2009;119:1420-8.

41. Feng J, Gou J, Jia J, Yi T, Cui T, Li Z. Verteporfin, a suppressor of YAP-TEAD complex, presents promising antitumor properties on ovarian cancer. Onco Targets Ther. 2016;9:5371-81.

42. Yu T, Cao G, Feng L. Low temperature induced de-differentiation of astrocytes. J Cell Biochem. 2006;99:1096-107.

43. Gram M, Sveinsdottir S, Ruscher K, Hansson SR, Cinthio M, Akerstrom B, et al. Hemoglobin induces inflammation after preterm intraventricular hemorrhage by methemoglobin formation. $\mathbf{J}$ Neuroinflamm. 2013;10:100.

44. Kamphuis W, Kooijman L, Orre M, Stassen O, Pekny M, Hol EM. GFAP and vimentin deficiency alters gene expression in astrocytes and microglia in wild-type mice and changes the transcriptional response of reactive glia in mouse model for Alzheimer's disease. Glia. 2015;63:1036-56.

45. Lee HH, Park SC, Choe IS, Kim Y, Ha YS. Time course and characteristics of astrocyte activation in the rat brain after injury. Korean J Neurotrauma. 2015;11:44-51.

46. Malatesta P, Appolloni I, Calzolari F. Radial glia and neural stem cells. Cell Tissue Res. 2008;331:165-78.

47. Barry DS, Pakan JM, McDermott KW. Radial glial cells: key organisers in CNS development. Int $\mathrm{J}$ Biochem Cell Biol. 2014;46:76-79.
48. Merkle FT, Tramontin AD, Garcia-Verdugo JM, Alvarez-Buylla A. Radial glia give rise to adult neural stem cells in the subventricular zone. Proc Natl Acad Sci USA. 2004;101:17528-32.

49. Sild M, Ruthazer ES. Radial glia: progenitor, pathway, and partner. Neuroscientist. 2011;17:288-302.

50. Faulkner JR, Herrmann JE, Woo MJ, Tansey KE, Doan NB, Sofroniew MV. Reactive astrocytes protect tissue and preserve function after spinal cord injury. J Neurosci: Off J Soc Neurosci. 2004;24:2143-55.

51. Diepenbruck M, Christofori G. Epithelial-mesenchymal transition (EMT) and metastasis: yes, no, maybe? Curr Opin Cell Biol. 2016;43:7-13.

52. Stone RC, Pastar I, Ojeh N, Chen V, Liu S, Garzon KI, et al Epithelial-mesenchymal transition in tissue repair and fibrosis. Cell Tissue Res. 2016;365:495-506.

53. Zhang FF, Morioka N, Kitamura T, Hisaoka-Nakashima K, Nakata Y. Proinflammatory cytokines downregulate connexin 43gap junctions via the ubiquitin-proteasome system in rat spinal astrocytes. Biochem Biophys Res Commun. 2015;464:1202-8.

54. Lei B, Sheng H, Wang H, Lascola CD, Warner DS, Laskowitz DT, et al. Intrastriatal injection of autologous blood or clostridial collagenase as murine models of intracerebral hemorrhage. J Vis Exp (JoVE) 2014;89:e51439.

55. Marinelli C, Di Liddo R, Facci L, Bertalot T, Conconi MT, Zusso $\mathrm{M}$, et al. Ligand engagement of toll-like receptors regulates their expression in cortical microglia and astrocytes. J Neuroinflamm. 2015;12:244.

56. Yan Y, Yu L, Castro L, Dixon D. ERalpha36, a variant of estrogen receptor alpha, is predominantly localized in mitochondria of human uterine smooth muscle and leiomyoma cells. PLOS ONE. 2017;12:e186078. 